\title{
Experimental evaluation of ILC applied to a six degrees-of-freedom industrial robot
}

\author{
Johanna Wallén, Mikael Norrlöf, Svante Gunnarsson \\ Division of Automatic Control \\ Department of Electrical Engineering \\ Linköpings universitet, SE-581 83 Linköping, Sweden \\ WWW: http://www. control.isy.liu.se \\ E-mail: johanna@isy.liu.se, mino@isy.liu.se, \\ svante@isy.liu.se
}

23rd October 2006

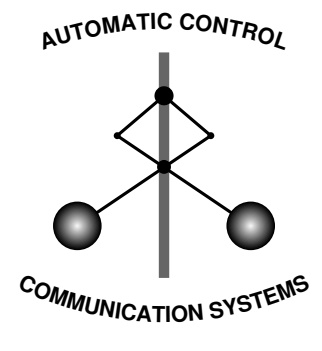

LINKÖPING

Report no.: LiTH-ISY-R-2750

Submitted to European Control Conference 2007 (ECC'07), Kos

Technical reports from the Control \& Communication group in Linköping are available at http://www.control.isy.liu.se/publications. 


\begin{abstract}
Experimental evaluation of an Iterative Learning Control (ILC) algorithm is presented. The ILC algorithm is applied on all six motors of a large size commercial six degrees-of-freedom industrial robot. The performance of the algorithm is evaluated with respect to the operating point of the robot, the programmed tool velocity, and the design variables of the ILC algorithm. The evaluated movements are intended to represent typical paths in a laser cutting application. Even though a fairly simple ILC algorithm is used the error reduction, evaluated using the measured motor angles, is substantial, and the algorithm shows good robustness properties.
\end{abstract}

Keywords: Iterative learning control, Mechanical systems/robotics 


\title{
Experimental evaluation of ILC applied to a six degrees-of-freedom industrial robot
}

\author{
Johanna Wallén, Mikael Norrlöf, and Svante Gunnarsson
}

\begin{abstract}
Experimental evaluation of an Iterative Learning Control (ILC) algorithm is presented. The ILC algorithm is applied on all six motors of a large size commercial six degreesof-freedom industrial robot. The performance of the algorithm is evaluated with respect to the operating point of the robot, the programmed tool velocity, and the design variables of the ILC algorithm. The evaluated movements are intended to represent typical paths in a laser cutting application. Even though a fairly simple ILC algorithm is used the error reduction, evaluated using the measured motor angles, is substantial, and the algorithm shows good robustness properties.
\end{abstract}

\section{INTRODUCTION}

A common application of industrial robots is to repeat the same series of events over and over again. The Iterative Learning Control (ILC) method is a way to compensate for a repetitive error when the same task is performed repeatedly. At every iteration the system starts from the same initial conditions and knowledge from previous iterations is used in order to reduce the error in next iteration.

The idea of using an iterative method to compensate for a repetitive error has been studied for some decades. When letting a machine do the same task repeatedly it is, at least from an engineering point of view, very sound to use knowledge from previous iterations of the same task to try to reduce the error next time the task is performed. The first academic contribution to what today is called ILC appears to be a paper by Uchiyama [24]. An application for a US patent on "Learning control of actuators in control systems" [10] was done already in 1967, and it was accepted as a patent in 1971. The idea in the patent was to store a "command signal" in a computer memory and iteratively update the command signal using the error between the actual response and the desired response of the actuator. This is clearly an implementation of ILC (see also [25]). From an academic perspective it was not until 1984 that ILC started to become an active research area. In 1984 [2], [6], and [8] were independently published describing a method that iteratively could compensate for model errors and disturbances. The development of ILC stems originally from the robotics area where repetitive motions show up naturally in many applications. Examples of contributions where ILC is applied in

This work was supported by the VINNOVA competence center ISIS and the Swedish Research Council (VR).

J. Wallén, S. Gunnarsson, and M. Norrlöf are with Division of Automatic Control, Department of Electrical Engineering, Linköping University, SE-58183 Linköping, Sweden, \{johanna, mino, svante\}@isy.liu.se robotics are [2], [4], [13], [14], [15], [9] and [23]. Examples of surveys on ILC can be found in [18], [7], [3] and [5]. In [17], [9] ILC is applied to a seven degrees-of-freedom robot arm. The manipulator used in [17], [9] is smaller than the one used here and the type of trajectories used in the experiments are not motivated by any particular application.

The simple and fast algorithm presented in this paper solves a relevant problem in laser cutting, and the purpose of this paper is to present results from extensive experiments carried out on all six motors of a large size commercial industrial robot. The experiments are performed using one of the larger robots from $\mathrm{ABB}$ with payload of around $175 \mathrm{~kg}$ accomplishing a small circular movement. Among the large number of publications dealing with various aspects of ILC there are very few publications presenting results of this kind, i.e., experimental evaluation using a large size commercial industrial robot where ILC is applied to all six motors.

\section{THEORETICAL BACKGROUND}

Consider the system depicted in Fig. 1. It is a system with four inputs: the reference signal $r(t)$, an externally generated control signal $u(t)$, and load and measurement disturbances $w(t)$ and $v(t)$, respectively. The measured output is $y(t)$ and the controlled variable is denoted $z(t)$. The system can have an internal feedback, which means that the blocks denoted $T_{u}, T_{r}, T_{w}$ and $T_{v}$ contain the system to be controlled as well as the control system in operation.

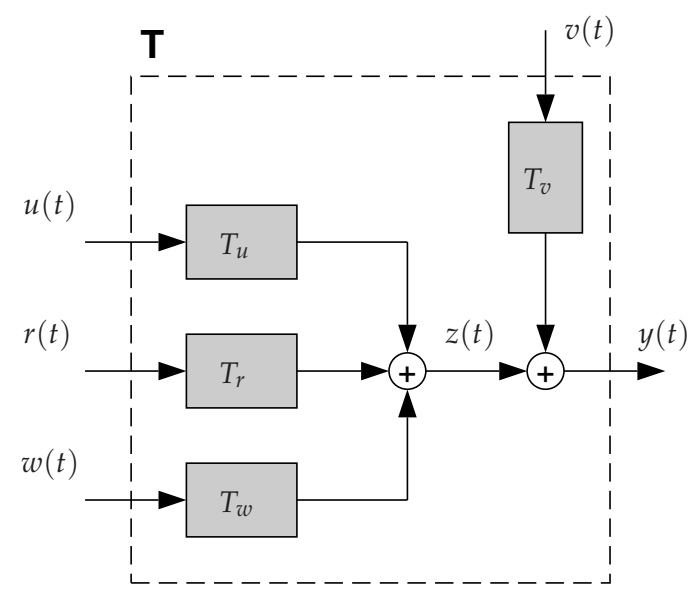

Fig. 1. System representation. 
In a situation where the system is both time and iteration invariant the system can be described using transfer operators

$$
\begin{aligned}
& z_{k}(t)=T_{r}(q) r(t)+T_{u}(q) u_{k}(t)+T_{w}(q) w_{k}(t) \\
& y_{k}(t)=z_{k}(t)+T_{v}(q) v_{k}(t) .
\end{aligned}
$$

The update equation for the first order ILC filter description with iteration independent operators is given by

$$
u_{k+1}(t)=Q(q)\left(u_{k}(t)+L(q) e_{k}(t)\right),
$$

where the subscript $k$ denotes the iteration number and $q$ is the time shift operator. The error

$$
e_{k}(t)=r(t)-y_{k}(t)
$$

is the difference between reference signal and measured output at iteration $k$. Applying the update equation (2) to the system (1) gives the standard frequency domain convergence criterion, see, e.g., [21],

$$
\left|1-L\left(e^{i \omega}\right) T_{u}\left(e^{i \omega}\right)\right|<\left|Q^{-1}\left(e^{i \omega}\right)\right|, \quad \forall \omega .
$$

The criterion shows that the filter $Q$ can be used to robustify the ILC algorithm. The inequality (4) can be satisfied by choosing the magnitude of the filter $Q$ small enough. It is well known that this will prevent the final error, after the ILC algorithm has converged, to be zero [9]. In [19] a time domain criterion for convergence is presented and a time varying filter $L$ is introduced, which gives monotonic decrease of the error as a function of iteration. Here a linear time invariant choice of filter $L$ will be utilized, and following the outline from [20] three main approaches to find $Q$ and $L$ can be found:

- Heuristic design.

- Model based frequency domain design.

- Model based time domain design using optimisation.

The paper [20] presents an extensive experimental comparison of the three approaches when applied to the first three degrees of freedom of a moderate size robot. One observation from this comparison is that the heuristic approach performs surprisingly well in comparison to the model based approaches. This is one reason for choosing this design method in the experiments presented below.

\section{PROBLEM DESCRIPTION}

\section{A. Robot system}

Fig. 2 shows an example of a large size industrial robot from $\mathrm{ABB}$, similar to the robot used in the experiments in this paper.

Note that the conventional robot controller, implemented by ABB in the IRC5 system, works in parallel with the ILC algorithm, i.e., ILC works as a complement to the conventional system and can be implemented without modifying the robot controller. The situation can schematically be depicted as in Fig. 3. As can be seen, the update $u_{k}(t)$ from the ILC algorithm in (2) is added to the reference signal.

All signals in the experiments correspond to the motor side, i.e., before the gearbox, since no measurements on the

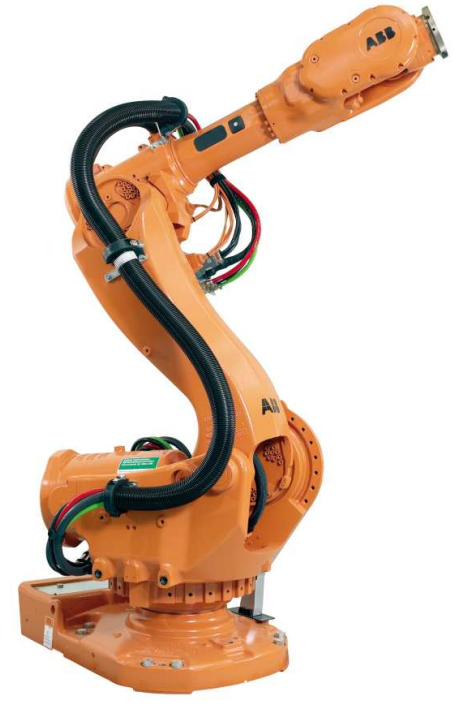

Fig. 2. Example of a large size industrial robot from ABB [1].

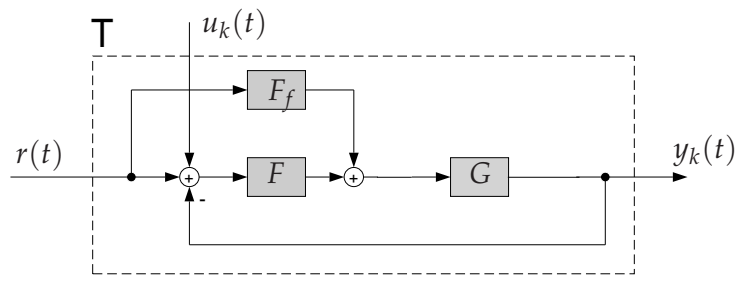

Fig. 3. Control system.

arm side are available. An analysis of the results on the arm side using a external measurement system, such as the Leica laser tracker [11], is left for future work.

In the experiments an interface between the robot control system IRC5 and MATLAB is used and the ILC algorithm is completely implemented in MATLAB.

\section{B. ILC algorithm}

The general ILC update equation, given in (2), is used in the experiments. As mentioned in Section II, a heuristic design procedure from [20] is used in this paper, which is formulated below.

1. Choose the $Q$ filter as a low-pass filter with cutoff frequency such that the bandwidth of the learning algorithm is sufficient.

2. Let $L=\gamma q^{\delta}$. Choose $\gamma$ and $\delta$ such that the stability criterion, formulated in the frequency domain $\left|1-L\left(e^{i \omega}\right) T_{u}\left(e^{i \omega}\right)\right|<\left|Q^{-1}\left(e^{i \omega}\right)\right|$, is satisfied. Normally it is sufficient to choose $\delta$ as the time delay and $0<\gamma \leq 1$ to get a stable ILC system.

$Q$ is according to the algorithm above chosen as a linear low-pass discrete time filter and $L$ is a linear discrete time filter $L(q)=\gamma q^{\delta}$, which can be non-causal. The control signal 
is then updated as

$$
u_{k+1}(t)=Q(q)\left(u_{k}(t)+\gamma e_{k}(t+\delta)\right),
$$

where the subscript $k$ denotes the iteration number.

The design variables in the ILC algorithm become:

- Type and order of filter $Q$.

- $Q$ filter cutoff frequency $\omega_{n}$.

- Gain $\gamma$, with $0.0<\gamma \leq 1.0$.

- Time shift $\delta$.

In the experiments presented in this paper the design variables $\omega_{n}$ and $\delta$ and their influence of the ILC algorithm performance are investigated. $Q$ is chosen as a second order butterworth filter, which is applied using the MATLAB function filtfilt in order to get a zero phase behaviour. The $L$ filter gain $\gamma$ is 0.9 during all the experiments and this is motivated by the trade-off between convergence rate and robustness.

\section{EXPERIMENTS}

The experiments have been performed in three different positions

$$
\begin{aligned}
& p_{1}=\left(\begin{array}{lll}
1.3166 & 0.0014 & 1.5992
\end{array}\right) \\
& p_{2}=\left(\begin{array}{lll}
1.8000 & 0.1000 & 1.5992
\end{array}\right) \\
& p_{3}=\left(\begin{array}{lll}
2.2000 & 0.2000 & 1.5992
\end{array}\right),
\end{aligned}
$$

which correspond to the tool position expressed in meters with respect to the robot base frame. The orientation quaternion of the tool is equal to

$$
q=\left(\begin{array}{llll}
0.6322 & 0.0353 & 0.7732 & 0.0353
\end{array}\right)
$$

in all the experiments. The robot configurations for the different positions are shown in Fig. 4. In each position the robot makes a circle of radius $5 \mathrm{~mm}$ with different velocities and filters $Q$ and $L$ in order to examine performance and robustness of the ILC algorithm. In each set of experiments one of the design variables in the ILC algorithm, see Section III-B, is varying while the others remain constant. Unless otherwise stated, the values used in the experiments are position $p_{1}$, velocity $v=50 \mathrm{~mm} / \mathrm{s}, Q$ filter cutoff frequency of $10 \mathrm{~Hz}$ and $L$ filter time shift $\delta=3$. In all experiments the same design parameters are used for all six motors. Since different dynamics can be expected for the motors connected to the three main axes compared to the motors connected to the tool orientation, it could be of interest to use different design parameters for the different groups of motors. This is however left for future work.

The results are presented as the norm of the control errors for each iteration, normalised with respect to the value without using ILC, according to

$$
\frac{\left\|e_{k, i, j}\right\|}{\max _{l, m}\left\|e_{0, l, m}\right\|},
$$

where $i=1, \ldots, 6$ (motor number), $j$ is experiment number and $k$ denotes the iteration. From a practical point of view the $\infty$-norm is the most relevant, since many applications require

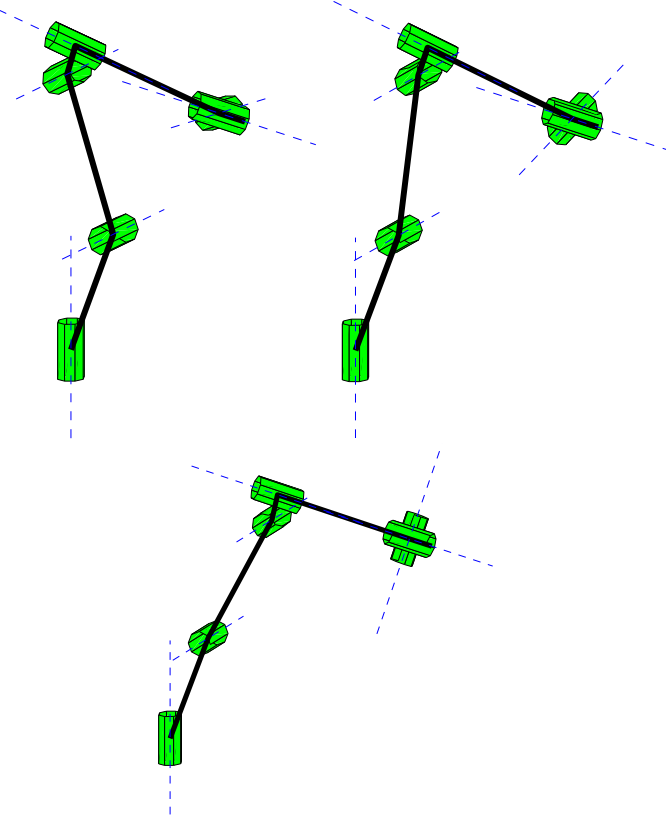

Fig. 4. The robot position, $p_{1}$ (top left), $p_{2}$ (top right), and $p_{3}$ (bottom).

a performance measure in terms of the maximum error. The convergence results in (4) are however given in 2-norm and therefore some results below are presented using this norm.

\section{A. Nominal performance}

In Fig. 5 the normalised control errors for the robot in the three positions (6) are shown. The size of the error changes significantly depending on position and motor. Motor 3 has the largest control error for position $p_{3}$, while motor 4 shows a large control error for position $p_{1}$ and $p_{3}$.
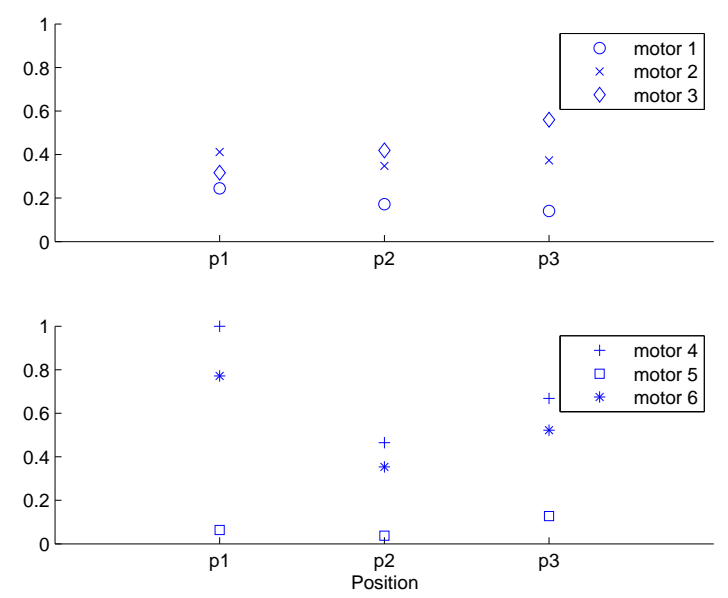

Fig. 5. The normalised control error before ILC iterations expressed in $\infty$-norm for three different positions $p_{1}, p_{2}$ and $p_{3}$.

The velocity dependent errors are shown in Fig. 6. As can be seen the normalised errors increase with increasing velocities, as expected. At high velocities the actual speed 
will not correspond to the programmed speed and this is the reason for the saturation of the error at $100 \mathrm{~mm} / \mathrm{s}$.
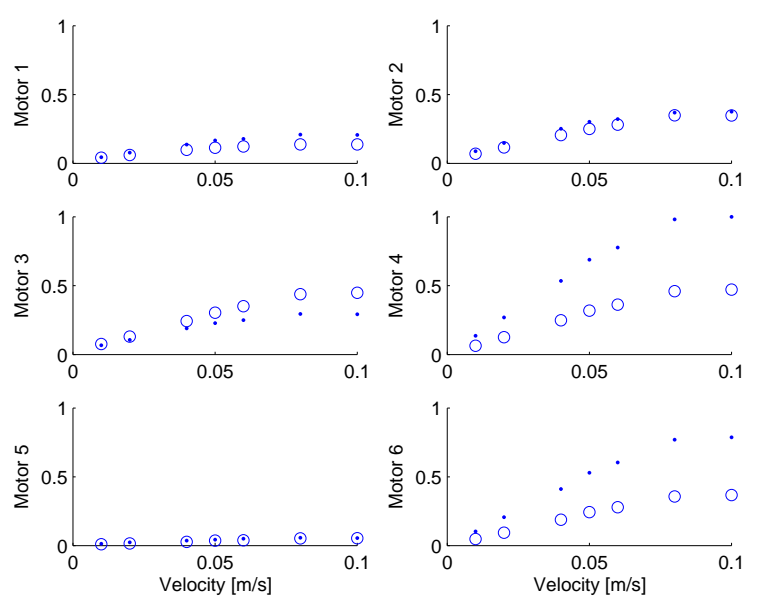

Fig. 6. The normalised control error before ILC iterations expressed in $\infty$-norm for different velocities from $0.01 \mathrm{~m} / \mathrm{s}$ to $0.10 \mathrm{~m} / \mathrm{s}$ and two different positions $p_{1}(\cdot)$ and $p_{2}(\circ)$.

\section{B. Stability test}

To examine the stability of the algorithm three different experiments are performed, one in each position $p_{1}, p_{2}$ and $p_{3}$. Fig. 7 shows how the normalised control error is decreasing as a function of iterations in position $p_{1}$. Similar results are achieved in position $p_{2}$ and $p_{3}$. The 2-norm of the error is used because if the algorithm is stable according to the convergence criterion (4), the error will decrease in this norm.

It can be seen that there are no significant changes of the errors after the fifth iteration. This motivates the choice to stop the learning after five iterations in the rest of the experiments. Future work should involve a more thorough investigation of the stability, for example including a better coverage of the full workspace of the robot.

The reduction of the error can be analysed in Cartesian space using the kinematic model of the robot. In Fig. 8 the motor reference, nominal robot performance $\left(y_{0}\right)$, and the trajectory achieved after three ILC iterations $\left(y_{3}\right)$ are shown. The experiment is performed with time shift $\delta=6$. The result in Fig. 8 indicates that the robot will follow the reference trajectory well also on the arm side. A complete evaluation would of course include measurements from the arm side, e.g., with a Leica laser tracker [11], but this is left for future work.

\section{Performance evaluation with different $L$}

Fig. 9 shows how different choices of time shift $\delta$ in the filter $L(q)=\gamma q^{\delta}$ affects the performance of the normalised error. It can be seen that there are only small differences in the control error when using different values of $\delta$. The case $\delta=0$ has not been included in the experiments since this corresponds to a causal ILC algorithm, see, e.g., [12], [22].
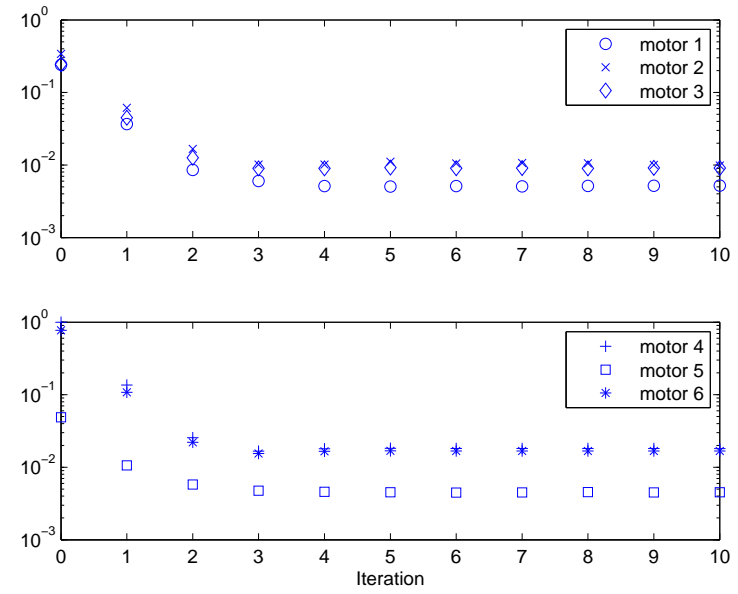

Fig. 7. The normalised control error for position $p_{1}$ expressed in 2-norm as a function of iterations.

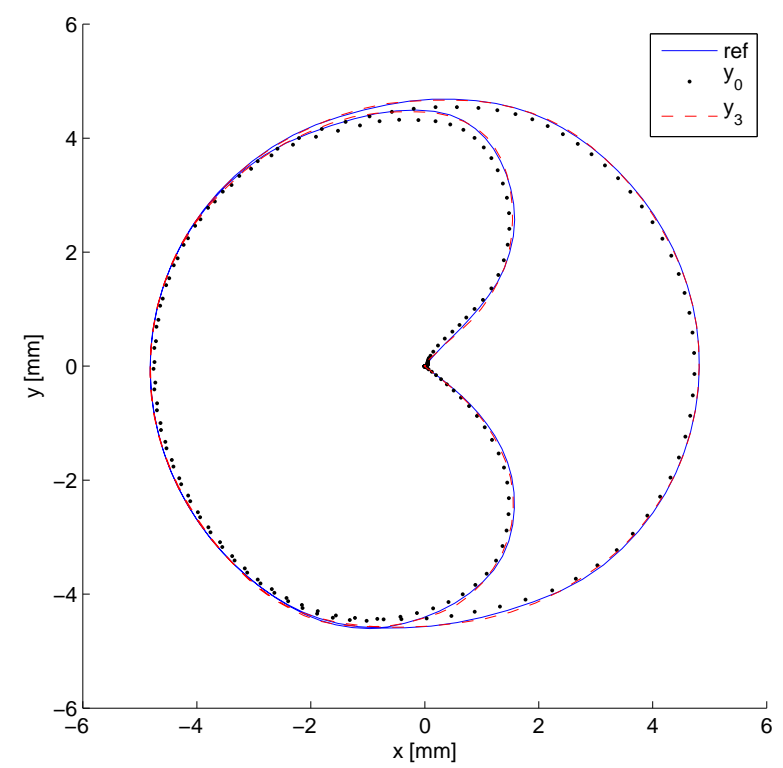

Fig. 8. The result after transforming the motor reference and measured positions to Cartesian space. The experiment is performed with $\delta=6$.

The model $T_{u}$, see (1), which describes the relation between $u_{k}(t)$ and $y_{k}(t)$, can be identified from experimental data. In this paper the model is identified using the System Identification Toolbox in MATLAB [16]. Input and output for the identification is created from the ILC experiments with different velocities (Section IV-D), according to

$$
u(t)=\sum_{v_{i}} u_{5, v_{i}}, \quad y(t)=\sum_{v_{i}} y_{5, v_{i}}-y_{0, v_{i}}
$$

where $u_{k, v_{i}}$ and $y_{k, v_{i}}$ is the ILC control signal and the output signal in iteration $k$ and experiment with velocity $v_{i}$. The validation data is created in the same way, but from another position. The best overall performance is achieved with an 

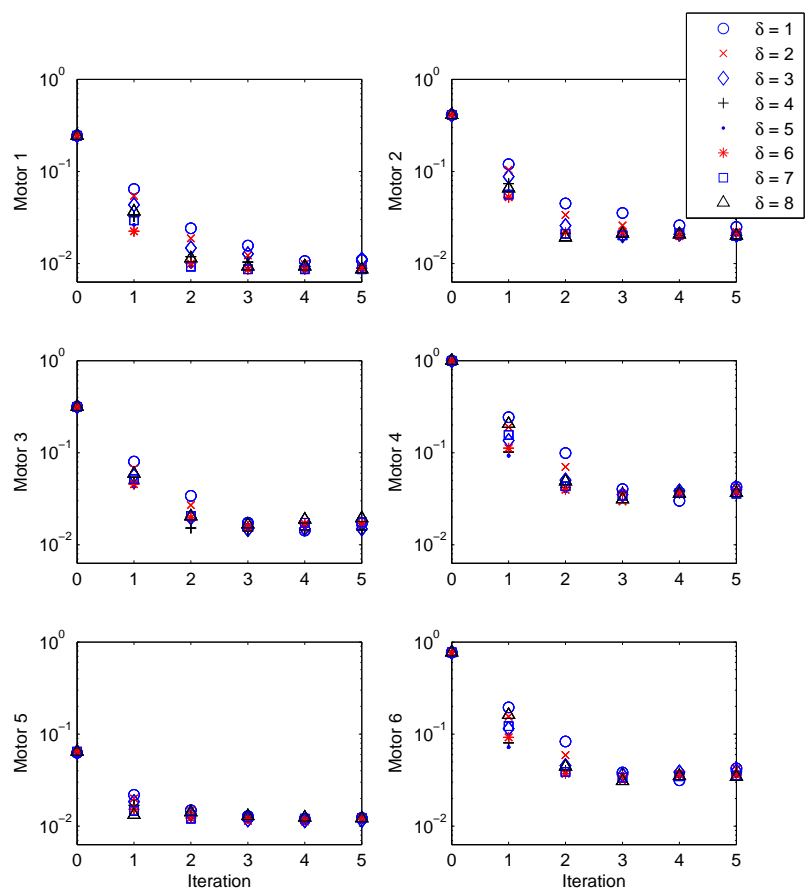

Fig. 9. The normalised control error for different choices of $\delta$ in the filter $L(q)=\gamma q^{\delta}$ as a function of iterations.

ARX model,

$$
T_{u}(q)=q^{-5} \frac{0.5083}{1-0.4884 q^{-1}} .
$$

In Fig. 10 the convergence criterion (4) for the ILC algorithm applied to the system is shown for $L$ filter time shift $\delta=1, \ldots, 8$. A gain as low as possible is desirable, and a gain higher than 1 gives a divergent behaviour. When having a low-pass filter $Q$, the ILC algorithm is robustified as described in Section II. In this paper $Q$ has a cutoff frequency of $5-22.5 \mathrm{~Hz}$, and in Fig. 10 the left hand side of the convergence criterion (4) is plotted with $\left|Q^{-1}\right|$ included for cutoff frequencies of $4 \mathrm{~Hz}, 10 \mathrm{~Hz}$, and $22.5 \mathrm{~Hz}$. The choice of $10 \mathrm{~Hz}$ will give a stable ILC algorithm for all choices of $\delta=1, \ldots, 8$, but for high cutoff frequencies $\delta=1,2$ will not satisfy the frequency domain criterion for stability in (4). Fig. 10 indicates that $\delta=6$ gives the highest suppression of the error for frequencies lower than $10 \mathrm{~Hz}$, which is the most relevant part of the frequency domain in this application. Compared to Fig. 9 it can be seen that a small time shift $\delta$ gives a slower convergence, according to the higher gain shown in Fig. 10.

\section{Performance evaluation at different velocities}

Fig. 11 shows the normalised control error expressed in $\infty$-norm when the robot performs the circle with different velocities and in two different positions $p_{1}$ and $p_{2}$, see (6). It can be seen that the errors are reduced with the same factor irrespective of the velocity. The steady state level changes

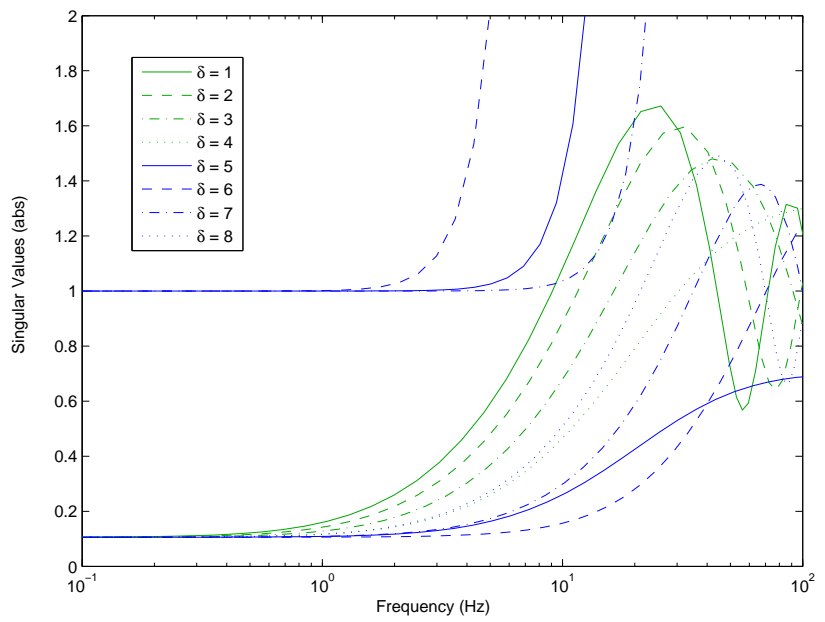

Fig. 10. Different choices of $\delta$ in the filter $L(q)=\gamma q^{\delta}$ affects the convergence properties of the normalised error. $\left|Q^{-1}\right|$ is shown for filter cutoff frequencies of $4 \mathrm{~Hz}$ (dashed), $10 \mathrm{~Hz}$ (solid), and $22.5 \mathrm{~Hz}$ (dashdotted).

because the frequency content of the error changes with the velocity. At low velocities most of the frequency content of the error is within the bandwidth of the ILC algorithm (see Fig. 10), while at higher velocities a more significant part of the error is outside of the bandwidth and the level of the steady state error increases. The small difference between $v=80 \mathrm{~mm} / \mathrm{s}$ and $v=100 \mathrm{~mm} / \mathrm{s}$ originates from the robot dynamic performance as explained as follows. The maximum velocity the robot can achieve in such a small circle is about $80 \mathrm{~mm} / \mathrm{s}$. Increasing the programmed velocity will not affect the error and this will be seen as a saturation of the error.

\section{E. Performance evaluation in different operating points}

Fig. 12 shows the normalised error in $\infty$-norm for three different positions $p_{1}, p_{2}$ and $p_{3}$. The control errors are reduced for all motors and in all positions. The low reduction of the error for motors 1 and 5 in $p_{3}$ is due to a high frequency error component which is outside of the bandwidth of the $Q$ filter in the ILC algorithm. It is left as future work to adjust the bandwidth of the $Q$ filter in this position to see if it is possible to reduce the effect of this disturbance on the error.

\section{F. Performance evaluation with different $Q$}

The cutoff frequency of the $Q$ filter directly affects the bandwidth of the ILC algorithm. In Fig. 13 the normalised error in $\infty$-norm using $Q$ filters with cutoff frequencies in the range $5-22.5 \mathrm{~Hz}$ is shown. The experiment is performed in position $p_{1}$. As expected a higher cutoff frequency gives a better reduction of the control error but as the cutoff frequency increases the error is no longer monotonically decreasing as a function of iteration. In Fig. 10 it is indicated that all the different choices of cutoff frequencies should 

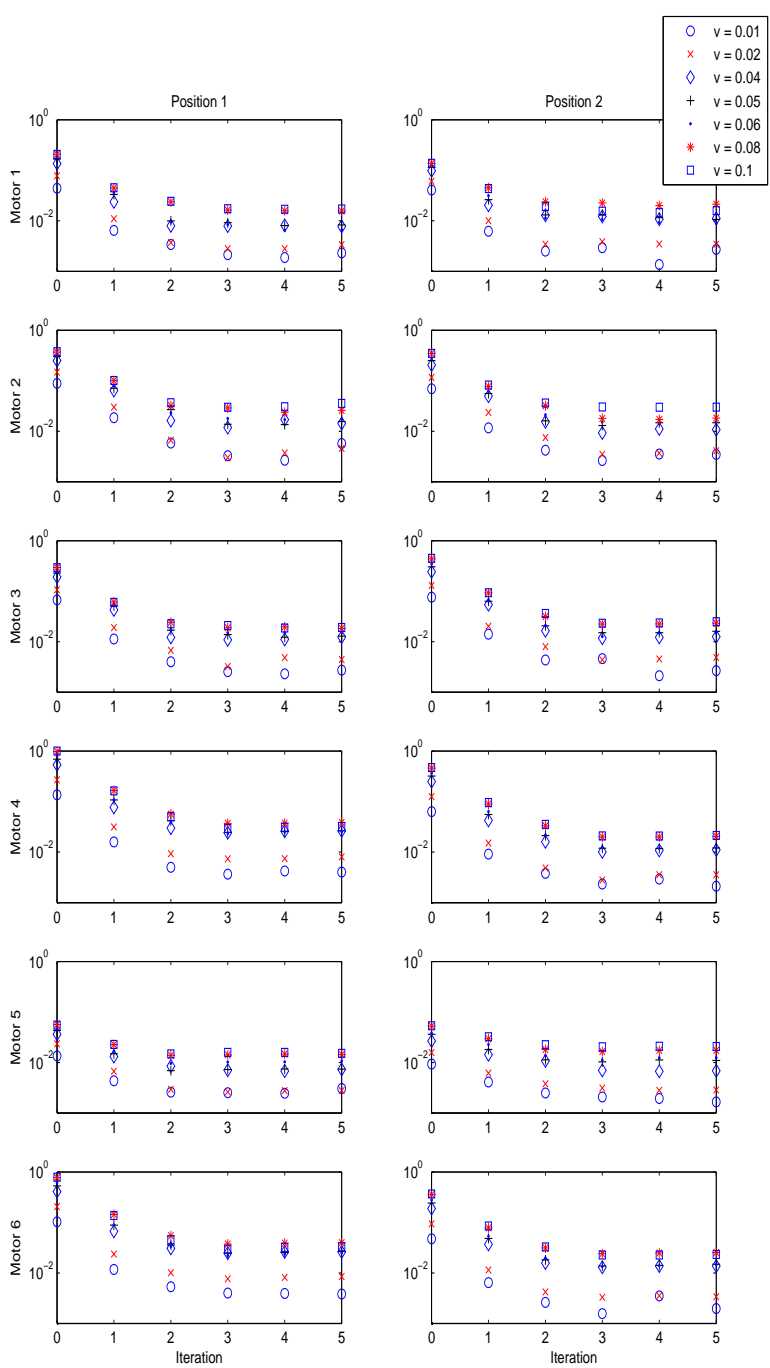

Fig. 11. The normalised control error expressed in $\infty$-norm for different velocities and two different positions $p_{1}$ and $p_{2}$ as a function of iterations.

result in monotonically decreasing 2-norm of the error for $\delta=3$ (actual value of $\delta$ in these experiments), but the model is uncertain at high frequencies and therefore the error can increase, as for motors 4 and 6. There are also disturbances, e.g., friction and backlash, that affect the errors in the different iterations.

The results in Fig. 13 shows that an increased bandwidth of the learning algorithm significantly improves the result for some motors, while for others the improvement is not that significant. Clearly this indicates that the same design parameters should not be used for all motors. An individual tuning of the parameters is left for future work.

\section{G. Repeatability}

Repeatability is examined by performing two identical experiments. The differences between the two experiments can
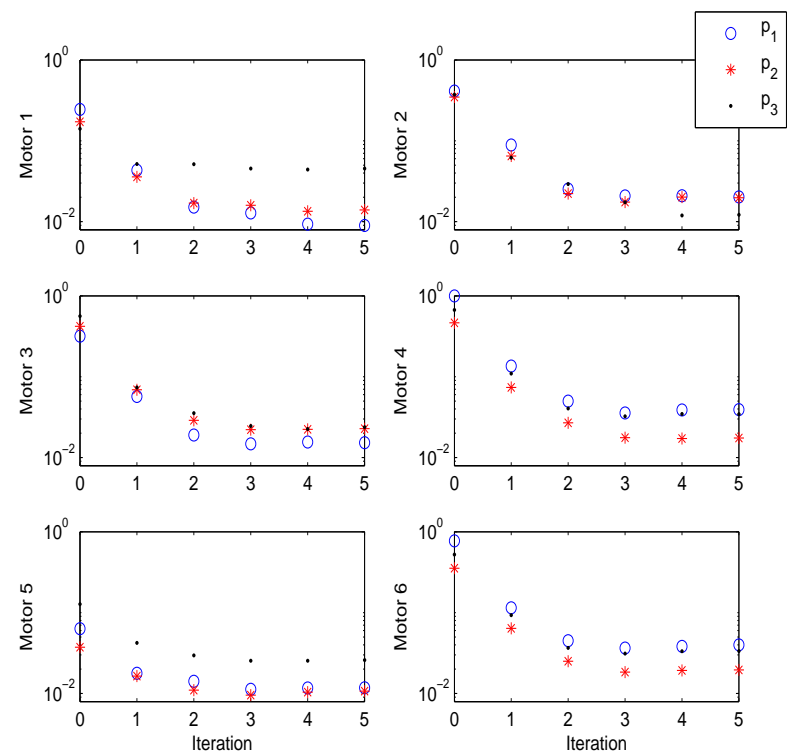

Fig. 12. The normalised control error expressed in $\infty$-norm for three different positions $p_{1}, p_{2}$ and $p_{3}$ as a function of iterations.
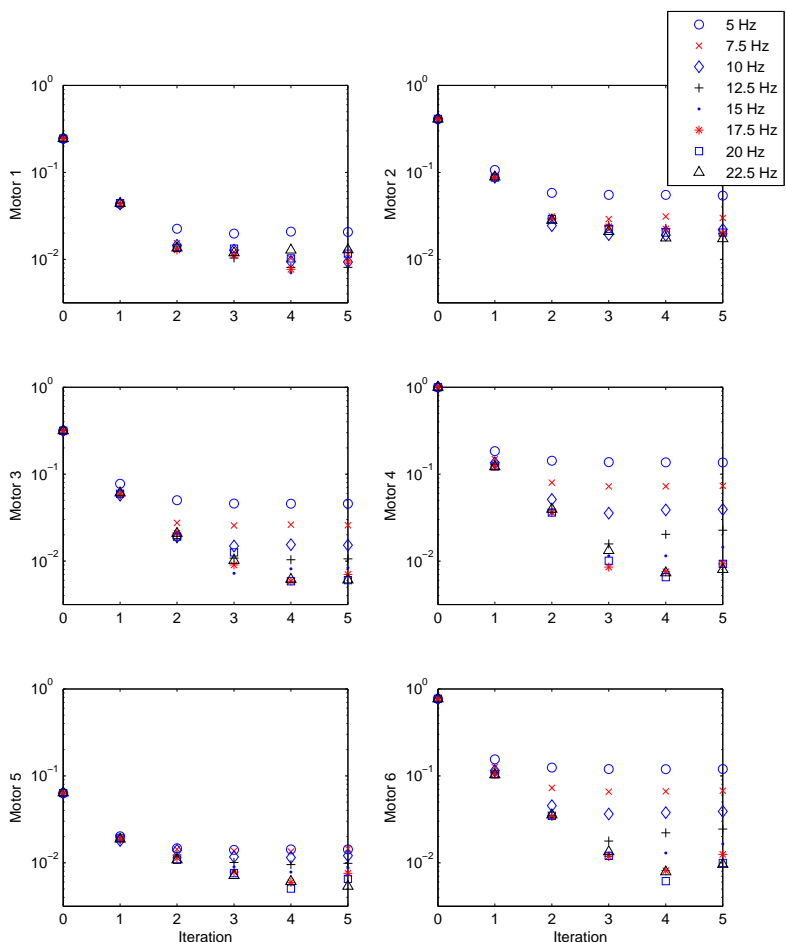

Fig. 13. The normalised control error in $p_{1}$ expressed in $\infty$-norm for butterworth filters $Q$ with different cutoff frequencies in the range from $5 \mathrm{~Hz}$ to $22.5 \mathrm{~Hz}$ as a function of iterations. 
be explained either by measurement disturbances or system disturbances, and the actual causes of the disturbances are not investigated further in this paper. The repeatability result is evaluated as the $\infty$-norm of the difference of the output for each iteration normalised with respect to the best value using ILC, according to

$$
\frac{\left\|y_{k, i, 1}-y_{k, i, 2}\right\|_{\infty}}{\min _{k, l, m}\left\|e_{k, l, m}\right\|_{\infty}}
$$

where $k$ denotes the iteration, $i=1, \ldots, 6$ (motor number), and $m$ is the experiment number. For the two experiments, the normalised repeatability error is about 0.2 , computed according to (11). The best that can be achieved is 1.0, which indicates that a more tailor-made ILC algorithm may reduce the control errors even more.

In order to be able to draw further conclusions, a larger set of identical experiments combined with a more thorough statistical analysis is needed.

\section{CONCLUSIONS AND FUTURE WORK}

An extensive experimental evaluation of a basic Iterative Learning Control (ILC) algorithm applied to all six motors of a commercial six degrees-of-freedom large size industrial robot has been presented. The performance with respect to design variables of the ILC algorithm and the operation point and velocity of the robot is examined. In all experiments the error has settled after approximately five iterations and thereafter the learning can be switched off. All results presented here are based on observations using measurements of the motor positions before the gearbox, and further work includes measuring the positions on the arm side, e.g., with a Leica laser tracker [11]. The results indicate that using different design variables for different motors may improve the results, this is however left for future work. Future experiments also include a larger set of identical experiments and a more thorough analysis of the repeatability. An important message in this contribution is that, although the ILC algorithm is very simple and basically only has two design parameters, time shift $\delta$ in the $L$ filter and cutoff frequency in the $Q$ filter, the error reduction is still very significant. The results also show that the algorithm is robust with respect to robot position and programmed velocity.

\section{ACKNOWLEDGMENTS}

This work was supported by the Swedish Research Council (VR) and the VINNOVA competence center ISIS.

\section{REFERENCES}

[1] ABB. Open public archive of robot images. www. abb. se, October 2006.

[2] S. Arimoto, S. Kawamura, and F. Miyazaki. Bettering operation of robots by learning. Journal of Robotic Systems, 1(2):123-140, 1984.
[3] Z. Bien and J.-X. Xu. Iterative Learning Control: Analysis, Design, Integration and Application. Kluwer Academic Publishers, Boston, MA, 1998.

[4] P. Bondi, G. Casalino, and L. Gambardella. On the iterative learning control theory for robotic manipulators. IEEE Journal of Robotics and Automation, 4:14-22, Feb 1988.

[5] D. A. Bristow, M. Tharayil, and A. G. Alleyne. A survey of iterative learning control. IEEE Control Systems Magazine, pages 96-114, 2006.

[6] G. Casalino and G. Bartolini. A learning procedure for the control of movements of robotic manipulators. In IASTED Symposium on Robotics and Automation, pages 108-111, San Francisco, USA, May 1984.

[7] Y. Chen and C. Wen. Iterative Learning Control: Convergence, Robustness and Applications, volume 248 of Lecture Notes in Control and Information Sciences. Springer-Verlag, 1999.

[8] J.J. Craig. Adaptive control of manipulators through repeated trials. In Proc. of ACC, pages 1566-1572, San Diego, CA, June 1984.

[9] H. Elci, R.W. Longman, M.Q. Phan, J.-N. Juang, and R. Ugoletti. Simple learning control made practical by zero-phase filtering: Applications to robotics. IEEE Transactions on Circuits and Systems I: Fundamental Theory and Applications, 49(6):753 - 767, June 2002.

[10] Murray Garden. Learning control of actuators in control systems. US Patent, US03555252, Jan 1971. Leeds \& Northrup Company, Philadelphia, USA.

[11] Leica Geosystems. Laser trackers, October 2006.

[12] Peter B. Goldsmith. On the equivalence of causal liti iterative learning control and feedback control. Automatica, 38:703-708, April 2002.

[13] K. Guglielmo and N. Sadegh. Theory and implementation of a repetetive robot controller with cartesian trajectory description. Journal of Dynamic Systems, Measurement, and Control, 118:15-21, March 1996.

[14] R. Horowitz, W. Messner, and J. B. Moore. Exponential convergence of a learning controller for robot manipulators. IEEE Transactions on Automatic Control, 36(7):890-894, July 1991.

[15] F. Lange and G. Hirzinger. Learning accurate path control of industrial robots with joint elasticity. In Proc. IEEE Conference on Robotics and Automation, pages 2084-2089, Detriot, MI, USA, May 1999.

[16] Lennart Ljung. System Identification Toolbox. User's Guide ver. 6.2. The MathWorks, 2006.

[17] R.W. Longman. Iterative learning control and repetitive control for engineering practice. International Journal of Control, 73(10):930 954, July 2000.

[18] K. L. Moore. Iterative learning control - an expository overview. Applied and Computational Controls, Signal Processing and Circuits, 1:151-214, 1999.

[19] K.L. Moore, Y. Chen, and V. Bahl. Monotonically convergent iterative learning control for linear discrete-time systems. Automatica, 41(9): 1529 - 1537, Septemeber 2005.

[20] M. Norrlöf and S. Gunnarsson. Experimental comparison of some classical iterative learning control algorithms. IEEE Transactions on Robotics and Automation, 18:636-641, 2002.

[21] M. Norrlöf and S. Gunnarsson. Time and frequency domain convergence properties in iterative learning control. International Journal of Control, 75:1114-1126, 2002.

[22] M. Norrlöf and S. Gunnarsson. A note on causal and CITE iterative learning control algorithms. Automatica, 41:345-350, 2005.

[23] A. Tayebi. Adaptive iterative learning control for robot manipulators. Automatica, 40(7):1195 - 1203, July 2004.

[24] M. Uchiyama. Formulation of high-speed motion pattern of a mechanical arm by trial. Trans. SICE (Soc. Instrum. Contr. Eng.), 14(6):706-712, 1978. Published in Japanese.

[25] Chen YuangQuang and Kevin.L Moore. Comments on us patent 3555252: Learning control of actuators in control systems. In ILC Invited Sessions at ICARCV'2000 (The Sixth International Conference on Control, Automation, Robotics and Vision). In ICARCV'OO CDROM, The Sixth International Conference on Control, Automation, Robotics and Vision, Singapore, December 5-8 2000. 


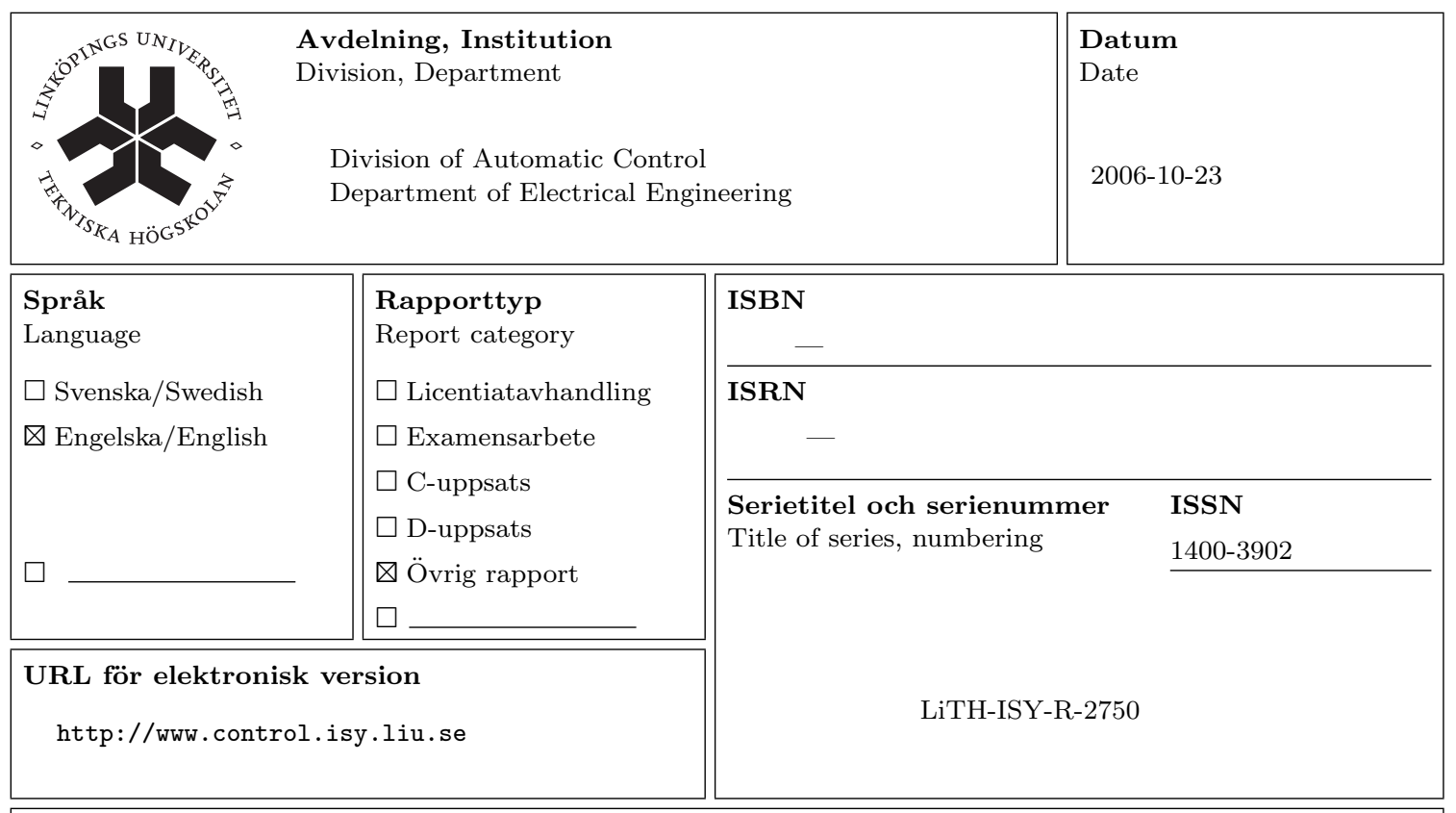

Titel Experimental evaluation of ILC applied to a six degrees-of-freedom industrial robot

Title

Experimental evaluation of ilc applied to a six degres-of-freedom industrial robot

Författare Johanna Wallén, Mikael Norrlöf, Svante Gunnarsson

Author

\section{Sammanfattning}

Abstract

Experimental evaluation of an Iterative Learning Control (ILC) algorithm is presented. The ILC algorithm is applied on all six motors of a large size commercial six degrees-of-freedom industrial robot. The performance of the algorithm is evaluated with respect to the operating point of the robot, the programmed tool velocity, and the design variables of the ILC algorithm. The evaluated movements are intended to represent typical paths in a laser cutting application. Even though a fairly simple ILC algorithm is used the error reduction, evaluated using the measured motor angles, is substantial, and the algorithm shows good robustness properties. 